\title{
Laser-assisted synthesis, and structural and thermal properties of ZnS nanoparticles stabilised in Polyvinylpyrrolidone
}

\author{
Damian C. Onwudiwe ${ }^{1}$, Tjaart P.J. Krüger ${ }^{2}$, Anine Jordaan, ${ }^{3}$ Christien A. Strydom ${ }^{1 *}$ \\ ${ }^{1}$ Chemical Resource Beneficiation (CRB) Research Focus Area, North-West University, Private Bag X6001, \\ Potchefstroom2520, South Africa \\ ${ }^{2}$ Department of Physics, University of Pretoria, Private Bag X20, Hatfield 0028, South Africa \\ ${ }^{3}$ Laboratory for Electron Microscopy, CRB Research Focus Area, North-West University, Private Bag X6001, \\ Potchefstroom 2520, South Africa \\ * Corresponding author: Prof. Christien A. Strydom \\ Telephone: +27182992340 \\ Fax: $\quad+27182992350$ \\ E-mail: $\quad$ christien.strydom@nwu.ac.za
}

\section{Research Highlights}

-Zinc sulphide (ZnS) nanoparticles were synthesised by laser irradiation

-The structural and morphological properties of the prepared samples were analysed

-Larger particles were obtained by using $\mathrm{Na}_{2} \mathrm{~S}$ instead of TAA as the sulphur source.

-Phonon softening and line broadening of the peaks were observed

-Size reduction occurred in the samples obtained from both sources 


\section{Graphical Abstract}
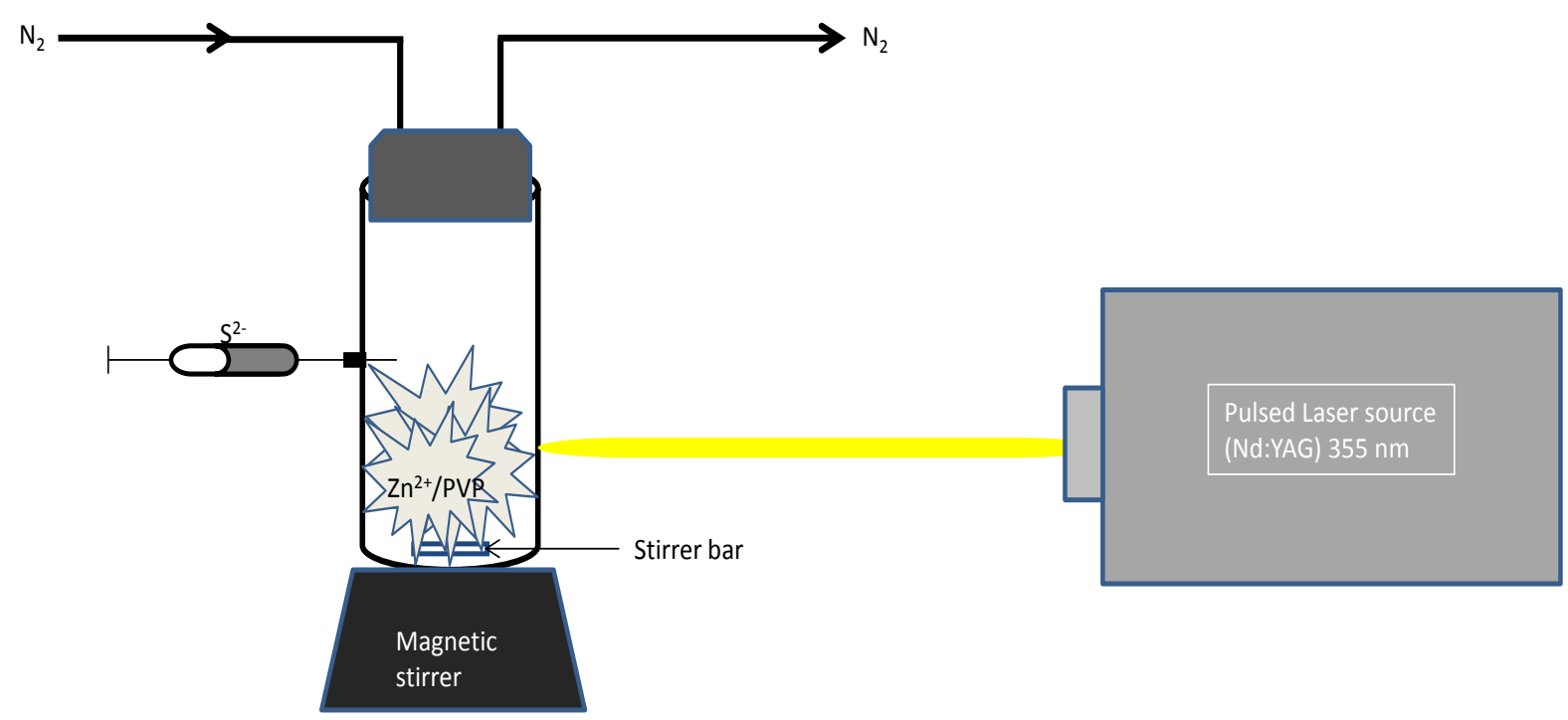

\section{Abstract}

Zinc sulphide (ZnS) nanoparticles have been synthesised by a green approach involving laser irradiation of an aqueous solution of zinc acetate $\left(\mathrm{Znac}_{2}\right)$ and sodium sulphide $\left(\mathrm{Na}_{2} \mathrm{~S} \cdot 9 \mathrm{H}_{2} \mathrm{O}\right)$ or thioacetamide (TAA) in polyvinylpyrrolidone (PVP). The structural and morphological properties of the prepared samples were analysed using a transmission electron microscope, TEM, a high resolution transmission electron microscope, HRTEM, X-ray diffraction, and Raman spectroscopy. The thermal properties were studied using a simultaneous thermal analyser (SDTA). Better dispersed and larger particles were obtained by using sodium sulphide $\left(\mathrm{Na}_{2} \mathrm{~S}\right)$ instead of TAA as the sulphur source. X-ray diffraction (XRD) analyses and Raman measurement show that the particles have a cubic structure, which is usually a low temperature phase of ZnS. There were phonon softening and line broadening of the peaks which are attributed to the phonon confinement effect. The average crystallite size of the ZnS nanoparticles estimated from the XRD showed a reduction in size from 13.62 to $10.42 \mathrm{~nm}$ for samples obtained from $\mathrm{Na}_{2} \mathrm{~S}$, and 9.13 to $8.16 \mathrm{~nm}$ for samples obtained from TAA, with an increase in the time of irradiation. The thermal 
stability of PVP was increased due to the incorporation of the ZnS nanoparticles in the matrices. The absorption spectra showed that the nanoparticles exhibit quantum confinement effects.

Keywords: Laser; ZnS nanoparticles; PVP capping; FTIR; Thermal stability; Raman spectra.

\section{Introduction}

The relationship between size and optical spectra was first established and confirmed for $\mathrm{ZnS}$ and CdS colloidal semiconductor nanoparticles (NPs) by Rosetti et al. [1-3]. Since then, numerous investigations have been carried out to synthesize colloidal semi-conductors (NCs), and the interest continues to increase [4-9]. Zinc sulphide is one of the important II-VI semi-conductors and has received wide attention. Its non-toxic nature and unique optical properties continue to increase its wide variety of applications [10]. Typical examples are in flat-panel displays, electroluminescence devices, IR windows [11, 12], sensors, lasers, and ultraviolet-emitting diodes $[13,14]$. Due to its non-toxicity, zinc sulphide is now used in waste water treatment as a photocatalyst in the degradation of organic pollutants such as dyes, halogenated derivatives, and $p$ nitrophenol [15-18]. ZnS can be prepared in various dimensions, such as isotropic dots, spheres, and anisotropic sheets, platelets, and diskettes [19-24].

The synthesis of nanoparticles using a green chemical route is an approach which has been receiving increasing attention [9]. Laser irradiation as a method of nanoparticle synthesis was initially introduced into materials chemistry in the synthesis of nanoparticles of noble metals via ablation techniques $[25,26]$, and this method has also been extended to the preparation of compound nanocrystalline materials $[27,28]$. Laser synthesis has the advantage of a high reaction rate and thus shorter reaction times. Due to synthesis difficulties such as Ostwald ripening and 
coalescence, which result in large particle sizes, and consequent loss of nanoparticulate properties, stabilizers are used to cap the surface layer of nanoparticles. The capping agents form a ligand shell around the nanoparticles, and apart from rendering the surface atoms passive, they also play the key role of determining the solubility of the nanoparticles in different solvents [29]. Surface passivation of nanoparticles by polyvinylpyrrolidone (PVP) has been investigated by many researchers [30, 31]. PVP (Figure 1), a water-soluble polymer, possesses the properties of a

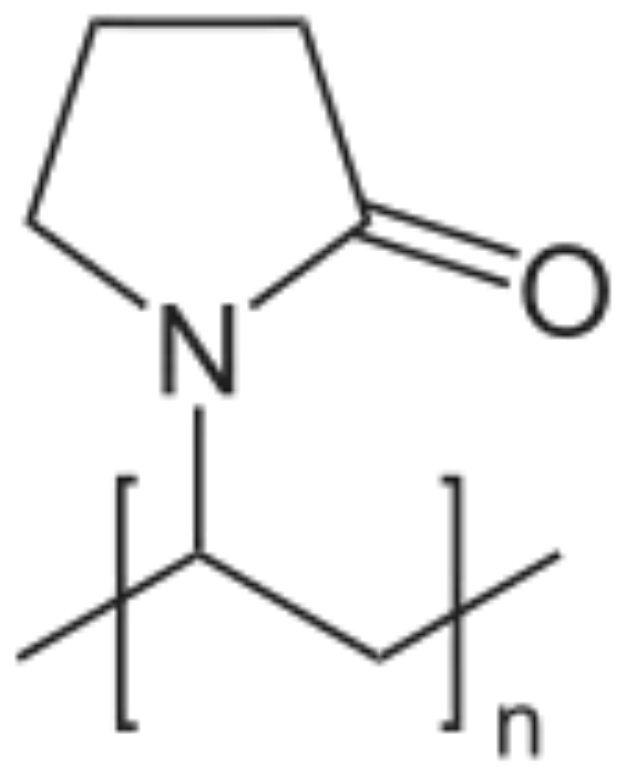

Fig. 1: Molecular structure of polyvinylpyrrolidone (PVP)

capping molecule. Typical of polymers, PVP acts as a ligand and forms a bond with the metal ions by donor acceptor interactions leading to the formation of a coordination sphere. In the polymer chain, the $\mathrm{N}$ and $\mathrm{O}$ atoms have lone pair of electrons which could be used in the formation of the bond [32]. The pyrrolidone (hydrophilic) part acts as the head group, while the polyvinyl (hydrophobic) part acts as the tail group. PVP controls the growth of the particles by forming passivation layers around the particle core via coordination bond formation. It, therefore, prevents agglomeration by steric effects due to the repulsive forces acting among the polyvinyl 
groups (tail part), thereby creating a restricted environment around the synthesised particles [33]. In our earlier studies [34], we have reported the solid state synthesis of ZnS and CdS nanoparticles. In this work, we report the room temperature synthesis of ZnS nanoparticles in an aqueous solution of PVP using laser irradiation. The main advantage of this method is that it permits larger quantities of nanoparticles to be produced at ambient temperature, and also in a short processing time. Two different sulphur sources where utilised and the structural properties of the nanoparticles where investigated.

\section{Experimental}

\subsection{Materials and Measurements}

Zinc acetate, polyvinylpyrrolidone, thioacetamide, and sodium sulphide nonahydrate were purchased from Merck, SA, and used as such without further purification.

The prepared samples were characterized by X-ray diffraction measurements on a Röntgen PW3040/60 X'Pert Pro X-ray diffractometer using Ni-filtered Cu K $\mathrm{K}_{\alpha}$ radiation $(\lambda=1.5405 \AA)$ at room temperature. For TEM observation, the samples were re-dispersed in ethanol by ultrasonic treatment and dropped on carbon-copper grids. Transmission electron microscopic (TEM) and high resolution transmission electron microscopic (HRTEM) images and selected area electron diffraction patterns were collected by using a TECNAI G2 ( $\mathrm{ACl})$ instrument operated at an accelerating voltage of $200 \mathrm{kV}$. The absorption measurements were carried out using a Perkin Elmer Lambda 20 UV-Vis spectrophotometer at room temperature. The thermal stability of the samples was studied using the simultaneous thermal analysis (SDTA) technique for parallel recording of TG (thermogravimetry) and DSC (differential scanning calorimetry) curves. The thermal investigations were performed on a SDTQ 600 Thermal analyser. Samples analysed were 
contained within alumina crucibles and heated at a rate of $10{ }^{\circ} \mathrm{C} \mathrm{min}-1$ under flowing nitrogen. Micro-Raman spectroscopy was performed with a HORIBA Jobin-Yvon T64000 spectrometer, using the $514.6 \mathrm{~nm}$ laser line of an Innova 70c Coherent argon-krypton mixed gas laser at an excitation power of $10 \mathrm{~mW}$. A $100 \times$ Olympus microscope objective was used to focus the laser beam tightly onto the top surface of one of the crystals of each sample. The spectra were acquired using five acquisitions of $120 \mathrm{~s}$ each.

The laser source used to synthesise the nanoparticles was an EKSPLA Nd:YAG nanosecond pulsed laser (NT342B-SH-10-AW), from which pulses with a peak wavelength of $355 \mathrm{~nm}$, duration of 3.6 $\mathrm{ns}$, and energy of $11 \mathrm{~mJ}$ were used at a rate of $10 \mathrm{~Hz}$. The beam diameter was $\sim 6.5 \mathrm{~mm}$ and was not notably changed by the sample cell (having a wall thickness of $1.5 \mathrm{~mm}$ and inner diameter of $25 \mathrm{~mm}$, the focal distance was one order of magnitude larger than the vessel diameter). This configuration delivered a peak intensity of approximately $150 \mathrm{~W} \mathrm{~cm}$ at the first quartz-water interface.

\subsection{Experimental Procedure}

Figure 2 presents a schematic diagram of the experimental set-up. In a quartz glass vessel (50 mL) containing $0.5 \mathrm{~g}$ polyvinylpyrrolidone in $10 \mathrm{ml}$ solution of de-ionised water, $0.25 \mathrm{M}$ zinc acetate ( $0.15 \mathrm{~g})$ was added and stirred continuously for $2 \mathrm{hr}$ to form a homogeneous mixture. Nitrogen gas was bubbled through the solution for $20 \mathrm{~min}$, and $0.30 \mathrm{M}$ sodium sulphide solution $(0.20 \mathrm{~g})$ was injected drop-wise into solution over a period of $10 \mathrm{~min}$. The reaction mixture was stirred for another 20 min to obtain a homogeneous stabilized dispersion. The flask containing the above mixture was subjected to laser irradiation for 30 or $60 \mathrm{~min}$. The precipitates were centrifuged and 
washed several times with distilled water and ethanol. The white products were dried under a decreased pressure atmosphere at room temperature. The same procedure was repeated using

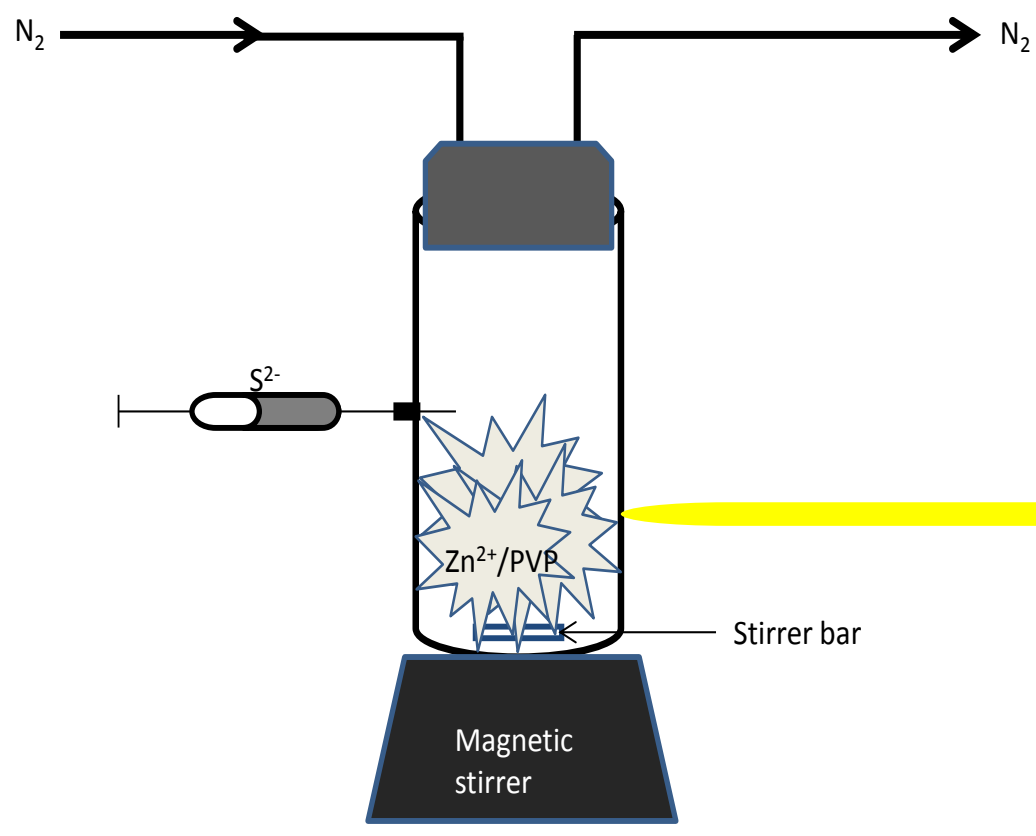

Pulsed Laser source (Nd:YAG) $355 \mathrm{~nm}$

Fig. 2: The schematic diagram of the experimental set-up.

TAA as sulphur source. The samples are denoted with $\mathrm{S} 1$ and $\mathrm{S} 2$, for products obtained from $\mathrm{Na}_{2} \mathrm{~S}$ after 30 and 60 min, respectively; and with S3 and S4 for products from TAA after 30 and 60 min, respectively.

\section{Results and discussion}

\subsection{Morphology and crystal structure of nanoparticles}

A transmission electron micrograph of samples S1 and S2, and their corresponding HRTEM images are shown in Figure 3. The Figure indicates that the morphology of the particles is roughly spherical and homogeneous. The particle size is approximately $10-12 \mathrm{~nm}$. The TEM images of samples S3 and S4 are presented in Figure 4, and the particles are quite small, with some degree of agglomeration, which hampers accurate size determination from the TEM. The change in the 
sulphur source affects the particles' morphology and dispersion, and could be attributed to the difference in structure and properties between TAA and $\mathrm{Na}_{2} \mathrm{~S}$.
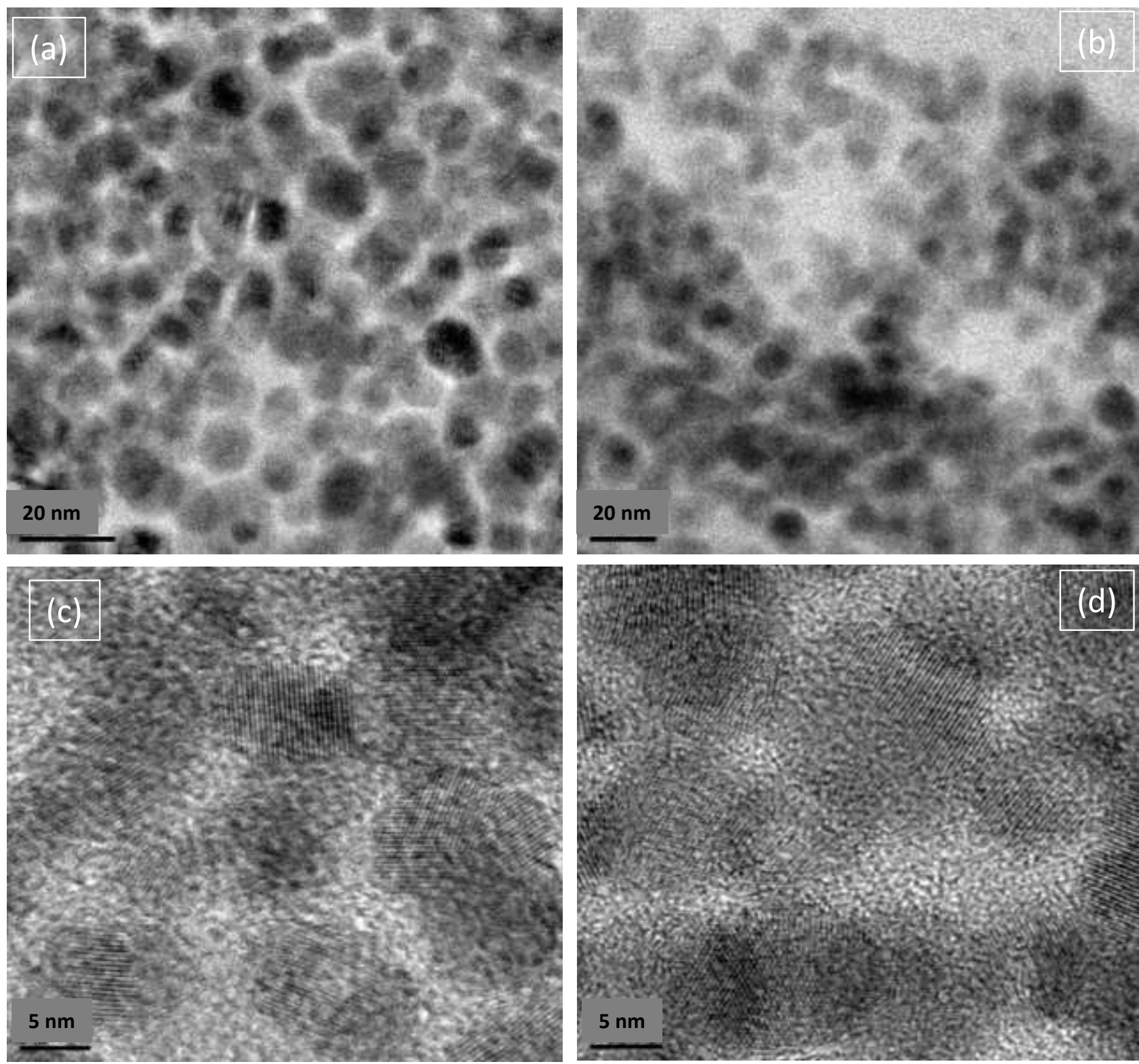

Fig. 3: TEM images of PVP stabilised $\mathrm{ZnS}$ samples prepared from $\mathrm{Na}_{2} \mathrm{~S}$ (a) after 30 min of laser irradiation (sample S1),

and (b) after 60 min of laser irradiation (sample S2), and the corresponding high resolution images (HRTEM) ((c) Sample S1 and (d) Sample S2) showing the lattice fringes. 

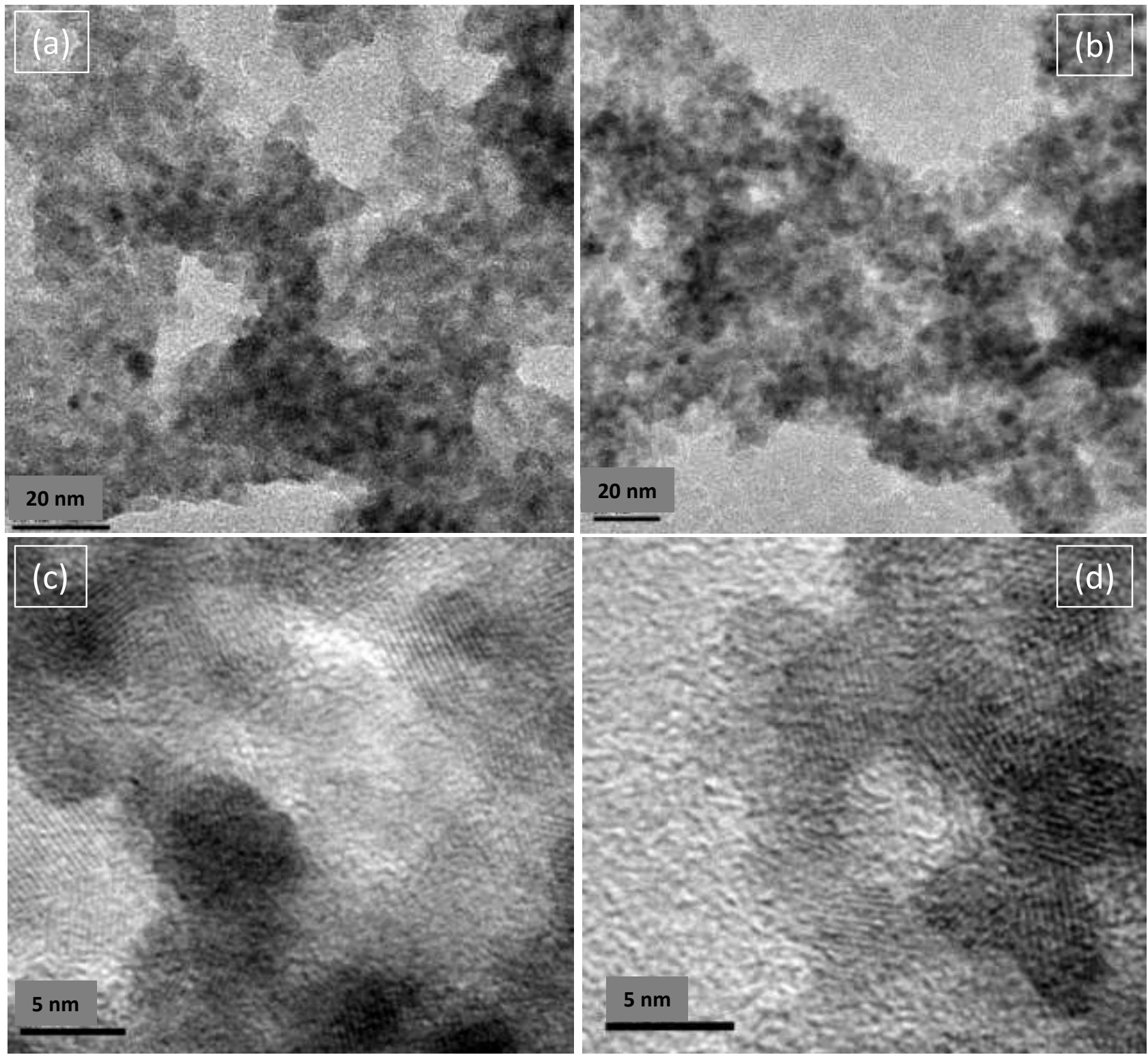

Fig. 4: TEM images of PVP stabilised ZnS samples prepared from TAA (a) after $30 \mathrm{~min}$ of laser irradiation (sample S3), and (b) after 60 min of laser irradiation (sample S4), and the corresponding high resolution images (HRTEM) ((c) Sample S3 and (d) Sample S4) showing the lattice fringes.

Figure 5 shows the HRTEM image together with FFT image (inset) taken from a single crystal of a ZnS sample prepared from $\mathrm{Na}_{2} \mathrm{~S}$ (a) and TAA (c) after 60 min of laser irradiation. The corresponding selected area electron diffraction patterns (SAED) ((b) for $\mathrm{Na}_{2} \mathrm{~S}$ and (d) for TAA) are also presented. The SAED patterns show a set of concentric rings instead of sharp spots indicating the presence of small crystallites. The diffraction rings correspond to reflections from three crystal planes, and can be assigned to the (111), (220), and (311) planes of cubic ZnS phase [35]. 

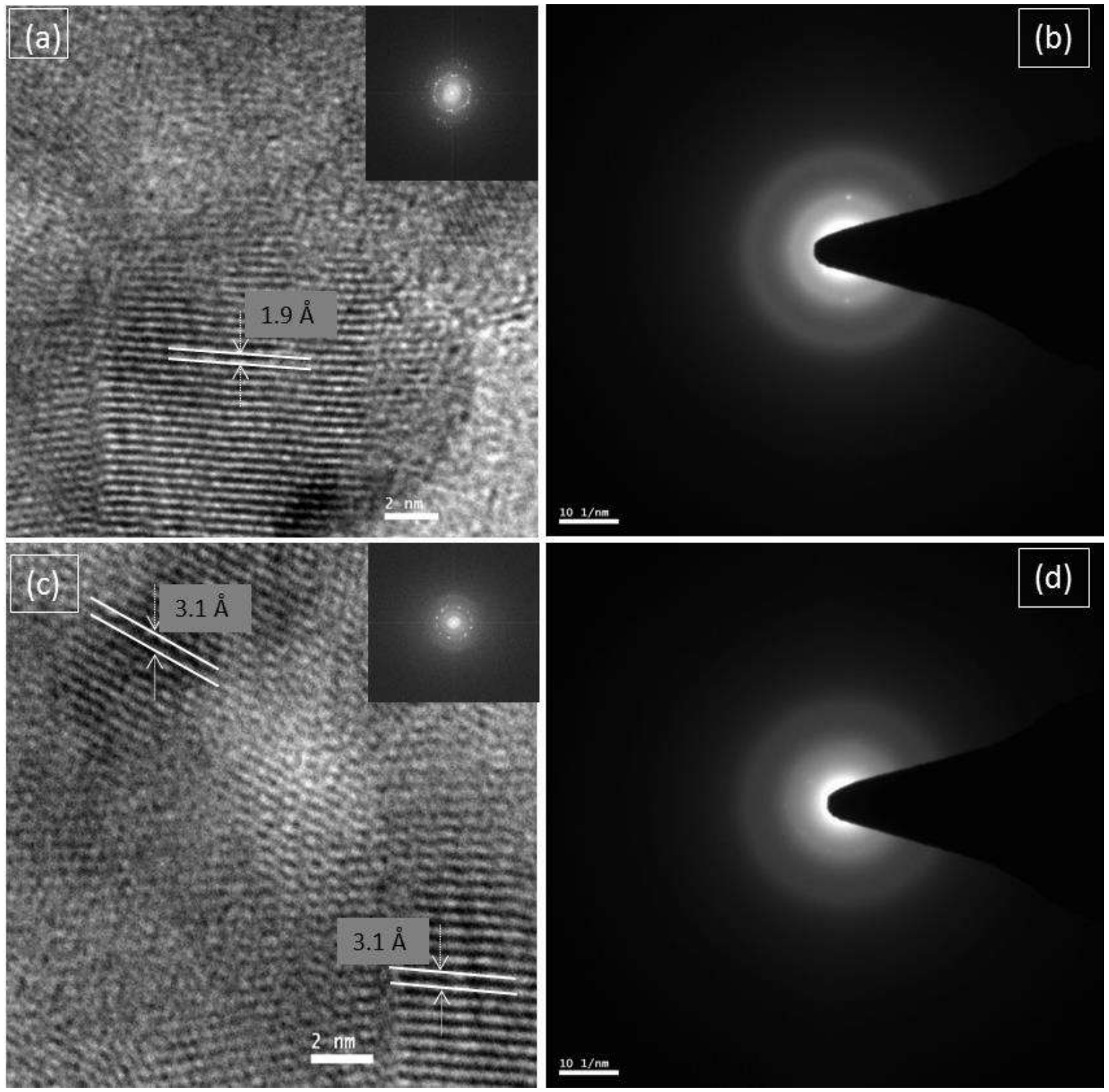

Fig. 5: HRTEM images of ZnS crystals formed from (a) $\mathrm{Na}_{2} \mathrm{~S}$, and (c) TAA, after 60 min of laser irradiation, with FFT (insets). (b) and (d) are the corresponding SAED patterns of crystalline ZnS. The crystals, which are aligned along the (111) axis with $d_{111}=0.31 \mathrm{~nm}$ and $d_{220}=0.19 \mathrm{~nm}$, are indicated.

X-ray diffraction provides an effective method for determining the phase and crystallite sizes of nanomaterial samples. Figure 6 shows the XRD patterns of the ZnS samples synthesised under various conditions. In all the samples, the peaks correspond to the (111), (220) and (311) planes of the cubic phase of ZnS according to JCPDS no. 04-5157. The particle sizes were estimated from the 


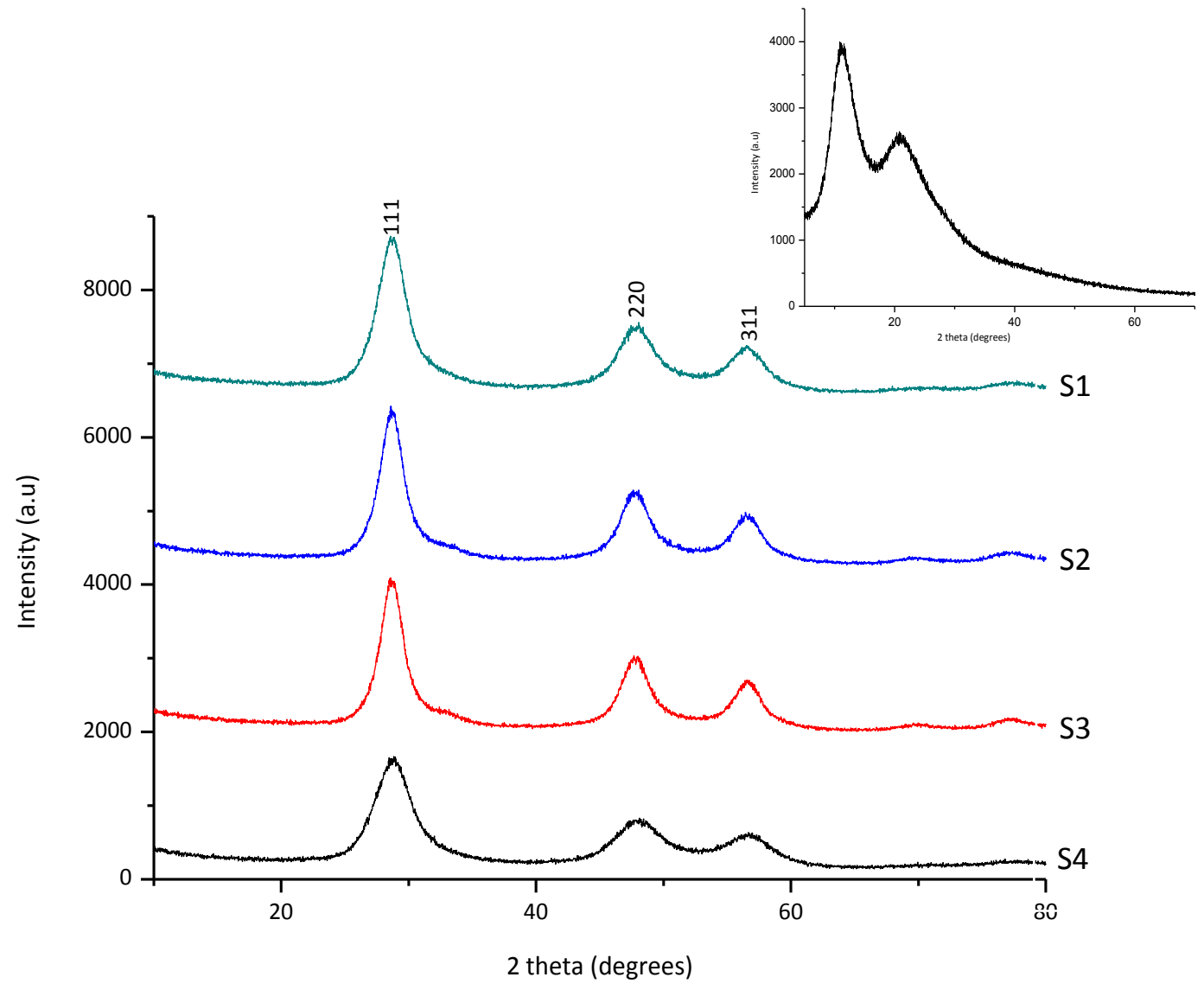

Fig.6: XRD patterns of samples prepared from $\mathrm{Na}_{2} \mathrm{~S}$ after $30 \mathrm{~min}(\mathrm{~S} 1), 60 \mathrm{~min}(\mathrm{~S} 2)$; from TAA after 30 min (S3) and after $60 \mathrm{~min}(\mathrm{~S} 4)$. The inset is the XRD pattern of pure PVP.

diffraction patterns based on the most intense (111) peak, by applying the Debye-Scherrer equation [36]:

$$
D=0.9 \lambda / \beta \cos \theta
$$

where $\lambda$ is the wavelength of the $\mathrm{Cu} K \alpha \mathrm{X}$-rays $(1.5405 \AA$ ) , $\beta$ is the FWHM of the diffraction peak corresponding to the (111) plane (in radians) and $\theta$ is the diffraction angle. Table 1 shows the particle sizes obtained from the products with different $\mathrm{S}^{2-}$ sources and at different reaction times. The particle sizes were found to be 10.4 - $13.6 \mathrm{~nm}$, and $8.4-9.1 \mathrm{~nm}$ for the samples prepared with $\mathrm{Na}_{2} \mathrm{~S}$ and TAA, respectively. A reduction in the particle size with increase in reaction time was 
Table 1: XRD characterization data of samples S1 - S4, with their average crystallite sizes estimated using the DebyeScherer's equation.

\begin{tabular}{|c|c|c|c|c|c|c|}
\hline Samples & 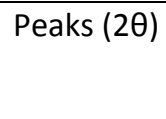 & d-spacings $(\AA)$ & $\mathrm{h}$ & k & 1 & $\begin{array}{l}\text { Average crystallite size } \\
(\mathrm{nm})\end{array}$ \\
\hline $\begin{array}{l}\mathrm{S} 1 \\
\left(\mathrm{Na}_{2} \mathrm{~S} \text { source, } 30 \mathrm{~min}\right)\end{array}$ & $\begin{array}{l}28.50 \\
47.38 \\
56.60\end{array}$ & $\begin{array}{l}3.132 \\
1.919 \\
1.625\end{array}$ & 2 & $\begin{array}{l}1 \\
2\end{array}$ & $\begin{array}{l}1 \\
0 \\
1\end{array}$ & 13.62 \\
\hline $\begin{array}{l}\mathrm{S} 2 \\
\left(\mathrm{Na}_{2} \mathrm{~S} \text { source, } 60 \mathrm{~min}\right)\end{array}$ & $\begin{array}{l}28.71 \\
47.65 \\
56.46\end{array}$ & $\begin{array}{l}3.109 \\
1.908 \\
1.629\end{array}$ & 2 & 2 & $\begin{array}{l}1 \\
0 \\
1\end{array}$ & 10.42 \\
\hline $\begin{array}{l}\text { S3 } \\
\text { (TAA source, } 30 \mathrm{~min} \text { ) }\end{array}$ & $\begin{array}{l}28.50 \\
47.96 \\
56.75\end{array}$ & $\begin{array}{l}3.129 \\
1.895 \\
1.621\end{array}$ & $\begin{array}{l}2 \\
3\end{array}$ & $\begin{array}{l}2 \\
1\end{array}$ & $\begin{array}{l}1 \\
0 \\
1\end{array}$ & 9.13 \\
\hline $\begin{array}{l}\text { S4 } \\
\text { (TAA source, } 60 \mathrm{~min} \text { ) }\end{array}$ & $\begin{array}{l}28.73 \\
48.16 \\
56.87\end{array}$ & $\begin{array}{l}3.107 \\
1.889 \\
1.619\end{array}$ & 2 & 2 & $\begin{array}{l}1 \\
0 \\
1\end{array}$ & 8.46 \\
\hline
\end{tabular}

observed. The reduction in size with reaction time is consistent with a report of the preparation of gelatin-stabilized silver nanoparticles via pulsed laser irradiation in solution [37]. For all the samples, there is a broadening of the peaks which is characteristic of nanosized particles. The inset in Figure 6 is the XRD pattern of the capping molecule (PVP). In the XRD patterns of the synthesized samples (S1 - S4), no peaks showing the presence of PVP are observed, which suggests that the PVP molecules are bonded onto the surface of the ZnS crystal rather than a solid mixture of ZnS and PVP being formed.

During the reaction, both the reactants and the products remain in the same solution. Thus, the synthesized nanoparticles receive further laser pulses with the same power as that which triggers their formation. This further interaction with laser pulses may be responsible for reduction in the particle sizes. During nanoparticle synthesis involving a solid phase in a liquid medium, reduction of nanoparticle sizes, resulting from the particle interaction with the laser beam, has been 
ascribed to the phenomenon of Coulomb or Thermal explosion in which large electron-free or deficient particles disintegrate into smaller ones [38]. The process of Coulomb explosion has so far only been described for metal nanoparticles in liquid medium, but the effect seems to also be occurring during this synthesis method of ZnS.

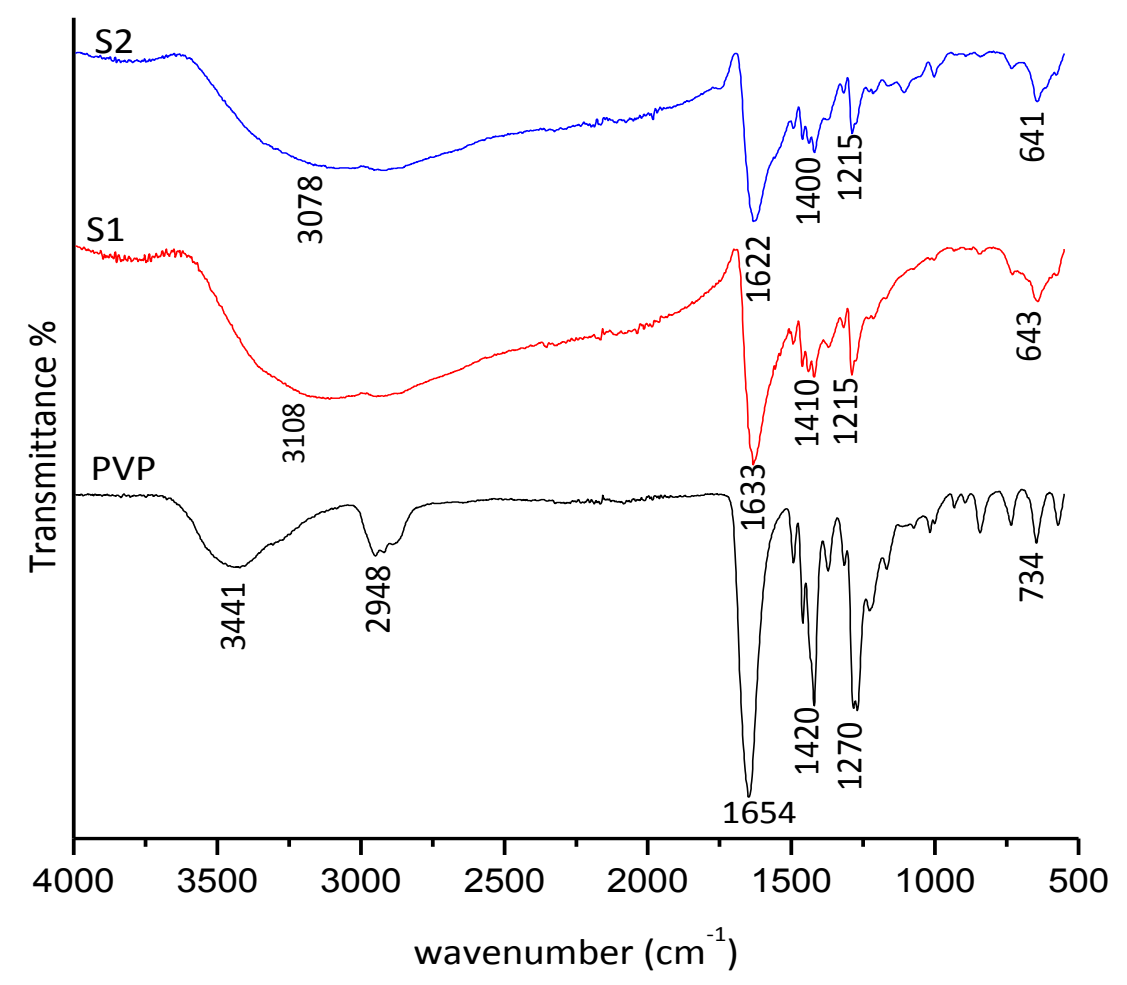

Fig. 7: FT-IR spectra of pure PVP (black), PVP-ZnS after 30 min laser treatment (S1, red), and PVP-ZnS after 60 min laser treatment (S2, blue).

\subsection{FT-IR spectroscopy}

Figure 7 presents the FT-IR spectra of pure PVP (bottom), PVP-ZnS after 30 min laser treatment (S1, middle), and PVP-ZnS after 60 min laser treatment (S2, top). For the pure PVP sample, the prominent absorption peak at $1654 \mathrm{~cm}^{-1}$ is due to the carbonyl group ( $C=0$ ) of PVP. This peak is observed to shift to $1633 \mathrm{~cm}^{-1}$ and $1622 \mathrm{~cm}^{-1}$ for samples S1 and S2, respectively. The shift in the vibrational frequency of $\mathrm{C}=\mathrm{O}$ is ascribed to the attachment of the polymer to the synthesized $\mathrm{ZnS}$ 
nanoparticles. A back-bonding occurred via the donation of the lone pairs of electrons on the oxygen atom of the carbonyl group to the $\mathrm{Zn}$ atoms leading to the weakening of the $\mathrm{C}=\mathrm{O}$ bond. The consequence is the passivation of the surface of the synthesized ZnS nanoparticles. The shift of this peak to lower wavenumber with reaction time could be ascribed to steric hindrance associated with the reduction in size of the ZnS nanoparticles.

In all the samples, a broad peak in the region $3300-3400 \mathrm{~cm}^{-1}$ is observed which is ascribed to O$\mathrm{H}$ stretching vibrations, and could be due to some adsorbed moisture [39]. The vibrational peak at around $2948 \mathrm{~cm}^{-1}$ in the pure PVP sample is due to $\mathrm{C}-\mathrm{H}$ vibrations. This peak is also observed to shift to lower wavenumber regions with increase in the reaction time, and overlaps with the broad $3300-3400 \mathrm{~cm}^{-1}$ band. The peaks at 641 and $643 \mathrm{~cm}^{-1}$ for samples S1 and S2 are assigned to the $\mathrm{Zn}-\mathrm{S}$ band. These peaks are absent in the spectrum of pure PVP.

\subsection{Raman spectroscopy}

Cubic $\mathrm{ZnS}$ belongs to the point group, $\mathrm{T}_{d}(43 \mathrm{~m})$, having two atoms per primitive unit cell and thus three optical phonons with $T_{2}=\Gamma_{15}$ symmetry. The optical modes, which may be easily observed by first-order Raman scattering, are doubly degenerate TO and single LO phonons with a higher frequency [40]. Figure 8 shows the Raman spectra of the ZnS samples. The Raman spectra of all the ZnS samples exhibit strong but broad peaks at $\approx 258$ and $342 \mathrm{~cm}^{-1}$. While the peak at $342 \mathrm{~cm}^{-1}$ is common to $\mathrm{ZnS}$ and is assigned to LO modes of the cubic ZnS, the strong peak at $258 \mathrm{~cm}^{-1}$ is not commonly observed. Kim et al. [41] have reported Raman spectroscopy of ZnS:Mn nanoparticles capped with mercapto-acetic acid, and assigned this peak to the TO modes. However, in the present study, we cannot assign this peak to the TO mode of cubic ZnS because the zone center and zone boundary TO phonon for bulk cubic ZnS are reported to appear at 276 and $303 \mathrm{~cm}^{-1}$, 


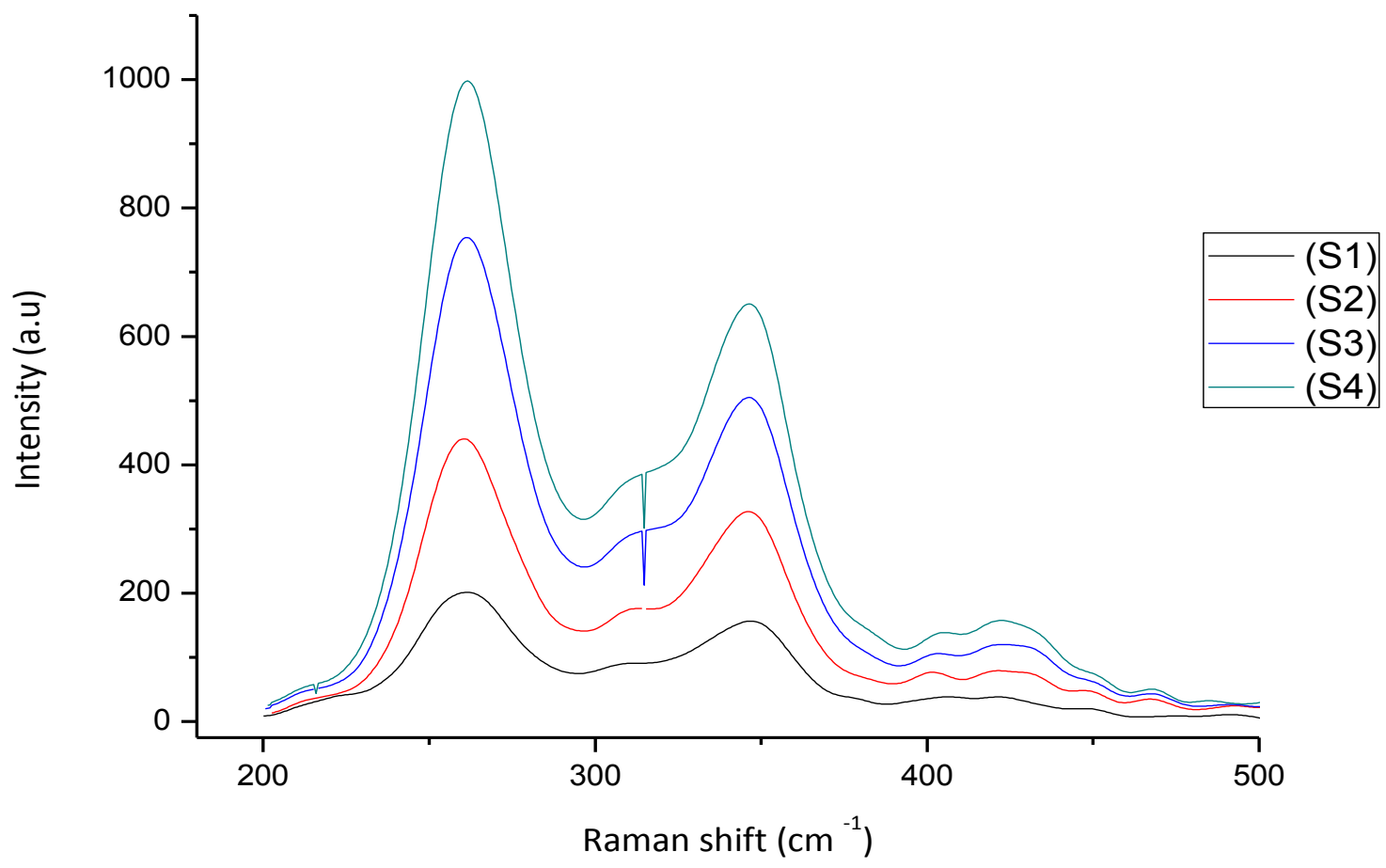

Fig. 8: Raman scattered spectra from PVP-ZnS samples prepared from $\mathrm{Na}_{2} \mathrm{~S}$ after 30 min laser treatment (S1), 60 min laser treatment (S2); from TAA after 30 min laser treatment (S3) and after 60 min laser treatment (S4).

respectively [42, 43]. Brafman and Mitra [44] reported the TO and LO zone center phonons of cubic ZnS crystals to be at 276 and $351 \mathrm{~cm}^{-1}$ respectively, for the Raman spectra of bulk cubic phases of ZnS. According to Smith [45], the coupling between longitudinal phonons and charge density fluctuations (plasmons) in polar semi-conducting systems results in two different LO phonon-plasmon coupled modes (LOPC modes), namely the $\mathrm{L}^{+}$and $\mathrm{L}^{-}$modes. As the carrier concentration in the semi-conductor increases, the frequency of the $\mathrm{L}^{+}$mode increases from bulk LO mode frequency and the frequency of $L^{-}$mode increases from zero to TO mode frequency [45]. Thus, in the present samples, the peak observed at $258 \mathrm{~cm}^{-1}$ can be attributed to the LOPC (L') mode. 
The absence of any peaks around $286 \mathrm{~cm}^{-1}$ in the Raman spectra of the prepared ZnS samples confirmed the phase of the samples to be cubic. In hexagonal phase ZnS, the Raman spectra exhibit peaks at 72 and $286 \mathrm{~cm}^{-1}$, which are ascribed to the E2 modes. Compared to the Raman spectra of bulk cubic ZnS, the first order LO phonon peak of the ZnS samples in the present study is shifted to lower frequency and the peak is asymmetrically broadened towards the lower frequency side. Such a phonon softening and line broadening of the peaks can be attributed to a phonon confinement effect [46]. In several semi-conductor materials, size-induced optical mode softening (red-shift) and acoustical mode hardening (blue-shift) effects are well known occurrences. Tensile and compressive strains affect the Raman spectra causing a red shift and a blue shift, respectively [47].

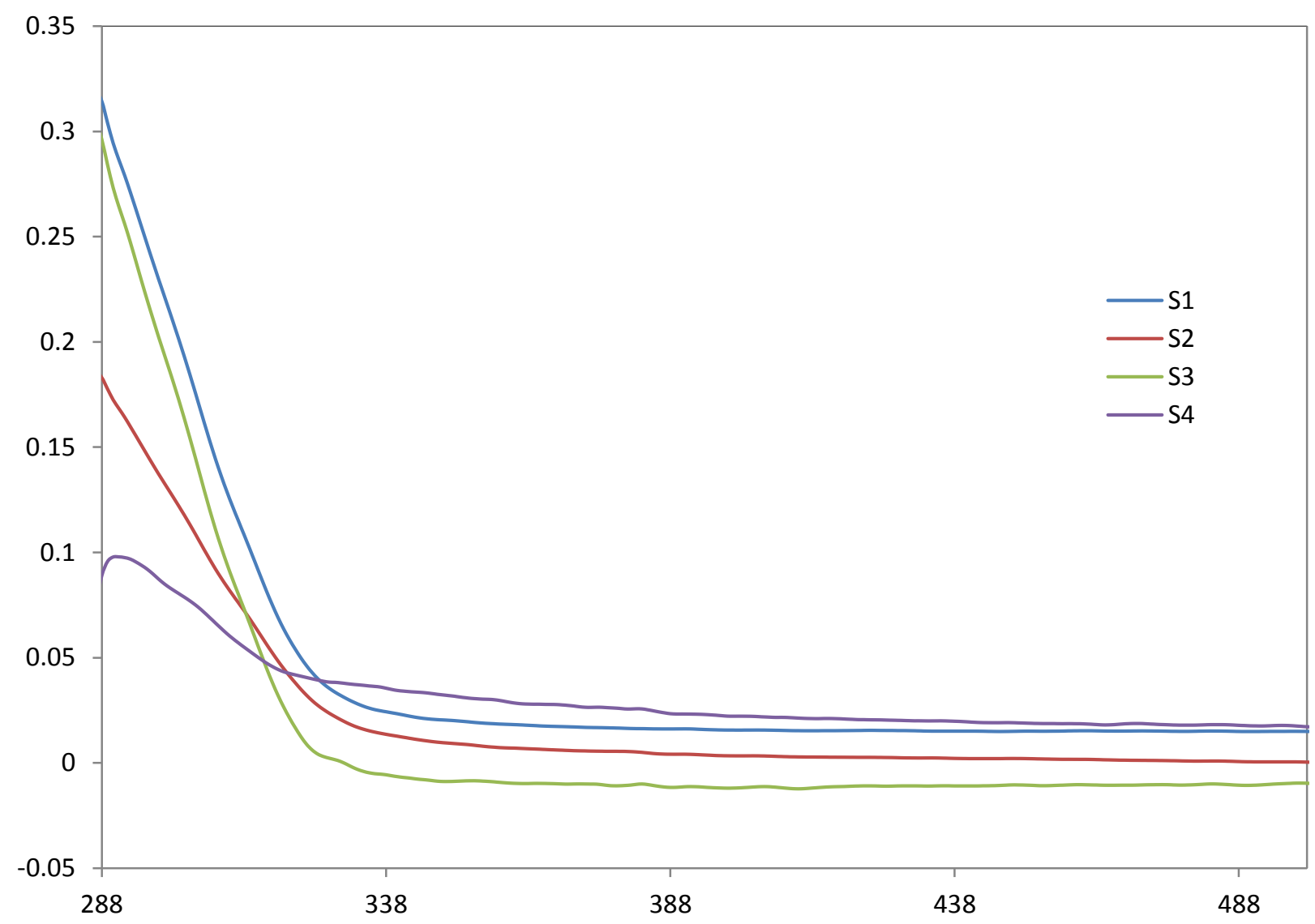

Fig. 9: UV-Vis absorption spectra of PVP stabilized ZnS nanoparticles prepared from $\mathrm{Na}_{2} \mathrm{~S}$ after 30 min laser treatment (S1), 60 min laser treatment (S2); from TAA after 30 min laser treatment (S3) and after 60 min laser treatment (S4). 


\subsection{Absorption spectroscopy}

UV-Vis spectra of the prepared ZnS nanoparticles (S1-S4) are shown in Figure 9. The spectra of all the samples showed a characteristic shift in the absorbance band edge to lower wavelength (320 $-330 \mathrm{~nm}$ ) relevant to the bulk materials. For cubic $\mathrm{ZnS}$ with a band gap of $3.54 \mathrm{eV}$ the absorbance edge is expected at a wavelength of $350 \mathrm{~nm}$. This observation has been ascribed to the phenomenon of quantum confinement effects [48].

\subsection{Thermal analysis}

The thermal properties of pure PVP as well as PVP-ZnS nanoparticles were examined by TGA to estimate how the thermal stability of PVP is affected by the ZnS nanoparticles. PVP thermally decomposes before melting. For comparison, the TG analysis of PVP was performed under the conditions similar to those used for the nanoparticle samples. The weight loss curve of pure PVP (Figure 10 (a)) shows a slight weight loss below $100^{\circ} \mathrm{C}$, which corresponds to an endothermic peak centred at approximately $83^{\circ} \mathrm{C}$. This peak is assigned to a glass transition $\left(T_{g}\right)$. Several conflicting values of the glass transition temperature $\left(T_{g}\right)$ of PVP are found in literature, and different suggestions to account for such differences include: presence of impurities, changes in specific heat involved, inability to attain near-equilibrium conditions during measurement and/or the faster rate of temperature change compared to the change in molecular rearrangement, along with the large influences of absorbed moisture due to the hygroscopic nature of the material [49]. A fast decomposition of the polymer chains of the PVP sample occurred between 378 and $487^{\circ} \mathrm{C}$ with a corresponding weight loss of $\approx 85 \%$ of the initial sample. A well-defined DTG peak centered at $428^{\circ} \mathrm{C}\left(T_{\max }\right)$ occurred with a DSC endothermic signal with maximum peak height at $430^{\circ} \mathrm{C}$. This is different from that of PVP-ZnS. Figure 10(b) obtained under similar experimental conditions shows the starting decomposition temperature to differ from that of the pure PVP sample. For 
(A)

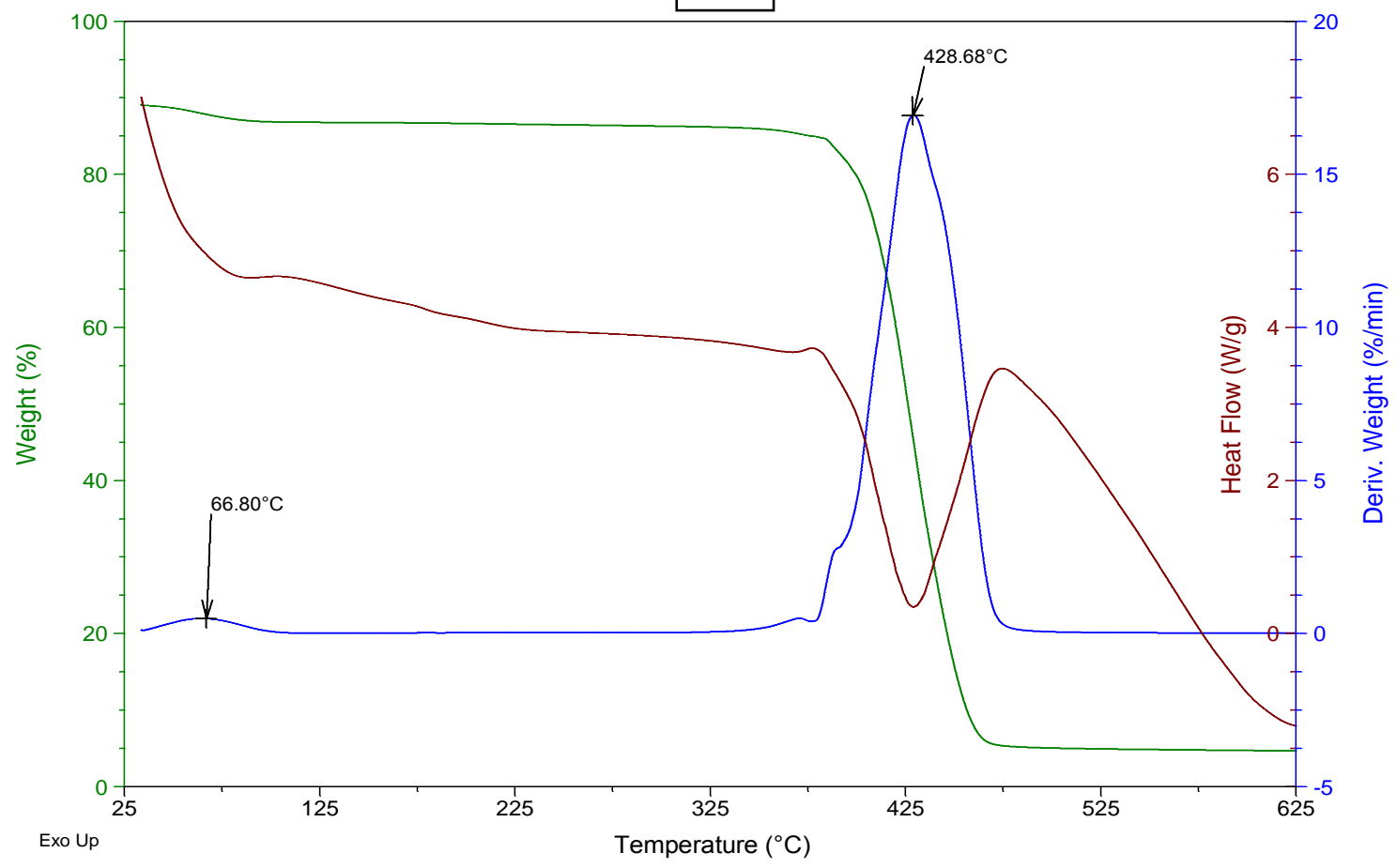

(B)

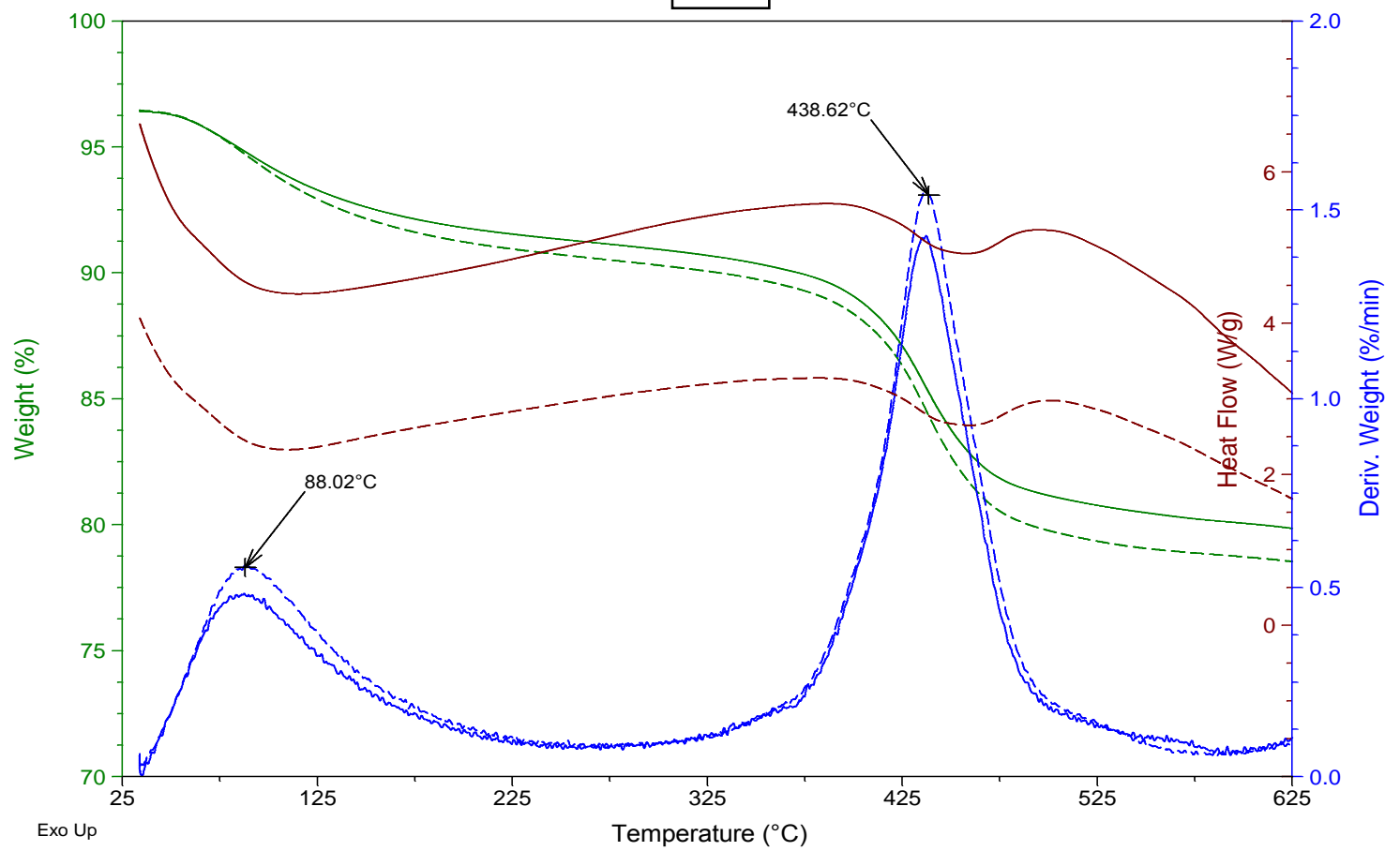

Fig. 10: TG, DTG and DSC curves of complexes (a) pure PVP, and (b) PVP-ZnS (solid line: sample S1, broken line: sample S2) obtained in nitrogen atmosphere $(75 \mathrm{~mL} / \mathrm{min})$, heating rate $10^{\circ} \mathrm{C} / \mathrm{min}$. 
PVP coated on ZnS nanoparticles, the DTG decomposition peak maximum is at $438^{\circ} \mathrm{C}, 10^{\circ} \mathrm{C}$ higher than that for pure PVP. This can be ascribed to the adsorption of the PVP molecules on the nanoparticle surface, which decreases the flexibility of the molecular chains, causing a nonequilibrium strained state of the polymer chain [50]. In comparison with pure PVP, three different thermal decomposition features for the polymer on the ZnS nanoparticle surface are observed: (1) the starting decomposition temperatures are higher; (2) the decomposition rate is slower; and (3) the relative heat flow change is smaller. This thermal behaviour concludes that the presence of ZnS in the PVP matrix does impart thermal stability to the resulting product.

\section{Conclusion}

ZnS nanoparticles stabilized in polyvinylpyrrolidone (PVP) with sizes between 8.5 to $13.6 \mathrm{~nm}$ were synthesised by a simple, fast, and less toxic synthesis method using laser energy. FT-IR spectroscopic measurements revealed the presence of PVP molecules on the surface of the nanoparticles. PVP plays an important role in enhancing the monodisperse property of the nanoparticles by the formation of coordinate bonds between the PVP and the $\mathrm{Zn}^{2+}$ ions, thereby lowering their surface energies. XRD and Raman spectra confirmed the phase of the samples to be cubic, and the observed red shift in the optical phonon modes is attributed to phonon confinement effects. The thermal stability analysis of PVP-ZnS showed that the presence of ZnS in the PVP matrix impart thermal stability to the resulting product. The quantum confinement effect is observed in the absorption properties of nanoparticles, and it is related to their small size. The presented method represents a green alternative to the conventional thermal decomposition synthesis method for ZnS nanoparticles, and can be extended to other II-VI semiconductor nanoparticles, such as CdS. 


\section{Acknowledgments}

This work was supported by National Research Foundation (NRF), South Africa, University of Pretoria, South Africa and North- West University, Potchefstroom, South Africa. Any opinion, findings and conclusions or recommendations expressed in this material are those of the author(s) and therefore NRF does not accept any liability in regard thereto.

\section{References}

[1] R. Rossetti, S. Nakahara, L. E. Brus. J. Chem. Phys. 79 (1983) 1086 - 1088.

[2] R. Rossetti, J. L. Ellison, J. M. Gibson, L. E. Brus. J. Chem. Phys. 80 (1984) 4464 - 4469.

[3] R. Rossetti, R. Hull, J. M. Gibson, L. E. Brus. J Chem. Phys. 82 (1985) 552 - 559.

[4] C.B. Murray, D.J. Norris, M.G. Bawendi, J. Am. Chem. Soc. 115 (1993) $8706-8715$.

[5] A.P. Alivisatos, Science. 271 (1996) 933 - 937.

[6] Z. A. Peng, X. Peng, J. Am. Chem. Soc. 123 (2001) 183 - 184.

[7] Y.A. Yang, H. Wu, K. R. Williams, Y.C. Cao, Angew. Chem. Int. Ed. 44 (2005) 6712 - 6715.

[8] N. Pradhan, D. Reifsnyder, R. Xie, J. Aldana, X. Peng, J. Am. Chem. Soc. 129 (2007) 9500 - 9509.

[9] R. Shahid, M. S. Toprak, M. Muhammed, J. Solid State Chem. 187 (2012) 130 - 133

[10] E. K. Goharshadi, R. Mehrkhah, P. Nancarrow, Mater. Scie. Semicon. Proc. 16 (2013) 356 - 362

[11] M. Bredol, J. Merichi, J. Mater. Sci. 33 (1998) 471 - 476.

[12] P. Calandra, M. Goffredi, V.T. Liveri, Colloids Surf. A (1601999) 9 - 13.

[13] T.V. Prevenslik, J. Lumen. 87-89 (2000) 1210 - 1212.

[14] T. Yamamoto, S. Kishimoto, S. lida, Physica B, 308-310 (2001) 916 - 919.

[15] Y. Wada, T. Kitamura, S. Yanagida, H. Yin, Chem. Commun. 24 (1998) 2683 - 2684.

[16] H. Fujiwara, H. Hosokawa, K. Murakoshi, Y. Wada,S. Yanagida, Langmuir 14 (1998) 5154 5159. 
[17] D. Chen, G. Shen, K. Tang, S. Lei, H. Zheng, Y. Qian, J. Cryst. Growth 260 (2004) 469 - 474.

[18] J. S. Hu, L. L. Ren, Y. G. Guo, H. P. Liang, A. M. Cao, L. J. Wan, C. L. Bai, Angew. Chemie. 117 (2005) $1295-1299$.

[19] H. Zhang, S.Y. Zhang, S. Pan, G.P. Li, J.H. Hou, Nanotechnology, 15 (2004) 945 - 948.

[20] S. Kar, S. Biswas, S. Chaudhuri, Nanotechnology, 16 (2005) $737-740$.

[21] Q. T. Zhao, L. S. Hou, R. A. Huang, Inorg. Chem. Commun. 6 (2003) $971-973$.

[22] Z.W. Pan, Z. R. Dai, Z. L. Wang, Science 291 (2001) 1947 - 1949.

[23] S. H. Yu, M. Yoshimura, Adv. Mater. 14 (2002) $296-300$.

[24] J. Q. Hu, Y. Bando, J. H. Zhan, Y. B. Li, T. Sekiguchi, Appl. Phys. Lett., 83 (2003) $4414-4416$.

[25] A. Takami, H. Kurita, S. Koda, J. Phys. Chem. B. 103 (1999) 1226-1232.

[26] S. Link, C. Burda, B. Nikoobakht, M. A. El-Sayed, J. Phys. Chem. B 104 (2000) 6152-6163.

[27] N. Kuthirummal, J. Reppert, B. Dihel, A.M. Rao, Appl. Opt. 48 (2009) $2842-2846$.

[28] F. Antolini, A. Ghezelbash, C. Esposito, E. Trave, L. Tapfer, B.A. Korgel, Mater. Lett. 60 (2006) $1095-1098$.

[29] D. Fan, M. Afzaal, M. Azad Mallik, C. Q. Nguyen, P.O’Brien, P. J. Thomas, Coord. Chem. Rev. 251 (2007) $1878-1888$.

[30] G. Murugadoss, V. Ramasamy, Spectrochim. Acta A, 93 (2012) $70-74$.

[31] T. T. Minh, B. P. Van, T. D. Van,H. N. Thi, Opt. Quantum Electron., 45 (2013) $147-159$.

[32] M. S. Khan, K. Gul, N. U. Rehman, Chin. J. Polym. Sci. 22 (2004) 581-584

[33] G. Ghosh, M. K. Naskar, A. Patra, M. Chatterjee, Opt. Mater. 28 (2006) 1047 - 1053.

[34] D. C. Onwudiwe, T.P.J. Kruger, C.A. Strydom, Mater. Lett. 116 (2014) $154-159$.

[35] Z. Shen, G. Chen, Q. Wang, Y. Yu, C. Zhou, Y. Wang, Nanoscale, 4 (2012) 2010-2017.

[36] E. Omurzak, T. Mashimo, S. Sulaimankulova, S. Takebe, L. Chen, Z. Abdullaeva, C. Iwamoto, Y. Oishi, H. Ihara, H. Okudera, A. Yoshiasa, Nanotechnology 22 (2011) $365602-365602$. 
[37] M. Darroudi, M. B. Ahmad, R. Zamiri, A. H. Abdullah, N.A. Ibrahim, A. R. Sadrolhosseini, Solid State Scie. 13 (2011) 520 - 524.

[38] R. R Letfullin, T. F George, in: Computational Studies of New Materials II: From Ultrafast Processes and Nanostructures to Optoelectronics, Energy Storage and Nanomedicine, 1999.

[39] N. Shanmugam, S. Cholan, N. Kannadasan, K. Sathishkumar, G. Viruthagiri, Solid State Scie. 28 (2014) $55-60$.

[40] Y. C. Cheng, C. Q. Jin, F. Gao, X. L. Wu, W. Zhong, S. H. Li, P. K. Chu, J. Appl. Phys. 106 (2009) $123505-123510$.

[41] J. E. Kim, C.-S. Hwang, S. Yoon, Bull. Korean Chem. Soc. 29 (2008) $1247-1249$.

[42] S. Saravana Kumar, M. Abdul Khadar, S.K. Dhara, T.R. Ravindran, K.G.M. Nair, Nucl. Instrum. Methods Phys. Res., Sect. B 251 (2006) $435-440$

[43] W.G. Nilsen, Phy. Rev. 182 (1969) 838 - 850.

[44] O. Brafman, S. S. Mitra, Phys. Rev. 171 (1968) 931 - 934.

[45] G.O. Smith, Phy. Rev. Lett. 68 (1992) 2366 - 2369.

[46] R. D. Yang, S. Tripathy, F. E. H. Tay, L. M. Gann, S. J. Chua, J. Vac. Sci. Technol. B 21 (2003) 984 $-988$.

[47] P. Nandakumar, C. Vijayan, M. Rajalakshmi, A.K. Arora, Y.V.G.S. Murti, Physica E 11 (2001) 377 $-383$.

[48] A.D. Yoffe, Adv. Phys. 42 (1993) 173-266.

[49] E. M. Abdelrazek, H.M. Ragab, M. Abdelaziza, Plastic and Polymer Tech. (PAPT) 2 (2013) 1 - 8.

[50] Y.S. Lipatov, L.M. Sergeeva. Adsorption of Polymers: Naukova Dumka, 1972. 